Z. Jagdwiss. 46 (2000), 128

() 2000, Blackwell Wissenschafts-Verlag, Berlin

ISSN 0044-2887

\title{
IV. REFERATE
}

\section{Referenten: BELGARD (Be.), HeNNIG (He.)}

\section{Wildkunde und Pathologie}

Finke, R.: Auf Tuch- und Borstenfühlung. Oberviechtach; Verlag Druckerei Forstner GmbH 1998. 128 S., 115 Abb., davon 74 Farbabb., geb. 49,80 DM. ISBN 3-00-003614-8.

Der Verfasser berichtet in flüssigem Plauderton chronologisch geordnet von den Erlebnissen, die er während sieben Jahren im Solling mit Schwarzwild der freien Wildbahn hatte. Durch unendliche Mühe und Geduld ist es ihm gelungen, führende Bachen und deren Frischlinge sowie Überläufer und sogar ältere Keiler so anzufüttern, dass sie sich anfassen ließen und zum Teil das Futter aus seinen sowie den Händen seiner Frau und seiner kleinen Tochter, vereinzelt sogar aus den Händen anderer begleitender Personen nahmen. Dabei konnte er viele interessante Verhaltensbeobachrungen machen. Von wissenschaftlichem Wert dürften vor allem Beobachrungen über Leitbachenfunktionen, Rangordnung und die wechselnde Zusammensetzung von Familienverbänden sein. Wenn an sich auch kein wissenschaftliches Werk, so eröffnet das Buch doch interessante Einblicke in das Leben des Schwarzwildes. Es liest sich sehr gut und besticht durch die Vielzahl hervorragender Photos.

He.

\section{Jagdpolitik und Jagdgesetzgebung}

NOLTE, M.: Die Erholungsfunktion des Waldes - Einfachgesetzliche Rechtsansprüche des Erholungsuchenden und ihre Grenzen unter besonderer Berücksichtigung des Straßenund Wegerechts, des Forst- und Waldrechts und des Naturschutzrechts-2. Aufl. Kiel; Jur. Diss. beim Lorenz-von-Stein-Institut für Verwaltungswissenschaften an der Universität Kiel (Arbeitspapiere; H 42) 1999. 225 S., DM 35,00.

Entsprechend der grundlegenden Zielsetzung werden die erholungsrelevanten Bestimmungen aus verschiedenen Rechtsgebieten systematisch analysiert und dogmatisch eingeordnet. Dazu werden die verfassungsrechtlichen Grundlagen sowie die bundeș- und landesrechtlichen, durch andere (z. B. kommunale) Normierungen ergänzten gesetzlichen Ausgestaltungen ausführlich untersucht. Dabei wird die Jagd, auch wenn ihr erholungbringende Momente für den Jagdausübenden selbst nicht abgesprochen werden, nicht der waldgesetzlichen Erholungsfunktion zugerechnet. Sie wird vielmehr zutreffend als eine die eigentlichen Erholungsakrivitäten deutlich beschränkende Nutzungsfunktion bewertet, was sich etwa in der Herausnahme der jagdbetrieblichen Einrichtungen aus dem allgemeinen Betretungsrecht zeigt.

Unter den begrenzenden Faktoren wird vor allem die Eigentumsgarantie des Art. 14 Abs. 1 GG als rechtliches Gegengewicht zu der allgemeinen Handlungsfreiheit dargestellt, das die Interessen unter anderem einer jagdwirtschaftlichen Nutzung vorbehaltlich der Sozialpflichrigkeir unterstützt. Hier wird auch auf die Möglichkeit einer wegen Jagdausübung aus ,wichtigem Grund ${ }^{\text {* }}$ gerechtfertigten privatnützigen Waldsperrung verwiesen, doch wird auf die vielfältigen Beeinträchtigungen der rechtmäßigen Jagdausübung durch eine übermäßige Inanspruchnahme des waldgesetzlichen Erholungsrechrs nicht eingegangen. Und insgesamt wird das Jagdrecht, das durch $\$ 3$ Abs. 1 BJG untrennbar mit dem Grundeigentum verbunden ist, nur verkürzt als gepachtetes Aneignungsrecht am herrenlosen Wild gesehen.

Nicht angesprochen werden auch die mit dem Jagdrecht gesetzlich verbundene Hegepflicht und ihr Ziel, einen artenreichen und gesunden Wildbestand zu erhalten und damit dazu beizutragen, dass der Wald überhaupt seine Erholungsfunktion erfüllen kann. Ebenso hätte neben den naturschutzrechtlichen Beschränkungen auch das Verbot des 19 a BJG erwähnt werden können, das Wild in bestimmten Bereichen unbefugt zu stören. Aber obwohl das Jagdrecht nach der Themenstellung nicht zu den Rechtskreisen gehört, die als das Erholungsrecht einschränkende gesetzliche Grenzen behandelt werden, enthält die Arbeit indirekt manche wichtigen Hinweise für den Jagdbetrieb. Daher kann sie durchaus eine Hilfe geben, wenn Konflikte zwischen jagdlichen Belangen und solchen der Erholungsuche zu lösen sind. 